\title{
Mass Description
}

National Cancer Institute

\section{Source}

National Cancer Institute. Mass Description. NCI Thesaurus. Code C90335.

A detailed account regarding the attributes of a body of matter. 